\title{
5
}

\section{Ground Reference Points in Legged Locomotion: Definitions, Biological Trajectories and Control Implications}

\author{
Marko B. Popovic \\ The Media Laboratory \\ Massachusetts Institute of Technology \\ U.S.A. \\ Hugh Herr \\ The MIT Media Laboratory \\ MIT-Harvard Division of Health Sciences and Technology \\ Spaulding Rehabilitation Hospital, Harvard Medical School
}

U.S.A.

The Zero Moment Point (ZMP) and Centroidal Moment Pivot (CMP) are important ground reference points used for motion identification and control in biomechanics and legged robotics. Using a consistent mathematical notation, we define and compare the ground reference points. We outline the various methodologies that can be employed in their estimation. Subsequently, we analyze the ZMP and CMP trajectories for level-ground, steady-state human walking. We conclude the chapter with a discussion of the significance of the ground reference points to legged robotic control systems. In the Appendix, we prove the equivalence of the ZMP and the center of pressure for horizontal ground surfaces, and their uniqueness for more complex contact topologies.

Since spin angular momentum has been shown to remain small throughout the walking cycle, we hypothesize that the CMP will never leave the ground support base throughout the entire gait cycle, closely tracking the ZMP. We test this hypothesis using a morphologically realistic human model and kinetic and kinematic gait data measured from ten human subjects walking at self-selected speeds. We find that the CMP never leaves the ground support base, and the mean separation distance between the CMP and ZMP is small (14\% of foot length), highlighting how closely the human body regulates spin angular momentum in level ground walking.

KEY WORDS-- Legged Locomotion, Control, Biomechanics, Human, Zero Moment Point, Center of Pressure, Centroidal Moment Pivot

\section{Introduction}

Legged robotics has witnessed many impressive advances in the last several decades-- from animal-like, hopping robots in the eighties (Raibert 1986) to walking humanoid robots at turn of the century (Hirai 1997; Hirai et al. 1998; Yamaguchi et al. 1999; Chew, Pratt, and 
Pratt 1999; Kagami et al. 2000). Although the field has witnessed tremendous progress, legged machines that demonstrate biologically realistic movement patterns and behaviors have not yet been offered due in part to limitations in control technique (Schaal 1999; Pratt 2002). An example is the Honda Robot, a remarkable autonomous humanoid that walks across level surfaces and ascends and descends stairs (Hirai 1997; Hirai et al.1998). The stability of the robot is obtained using a control design that requires the robot to accurately track precisely calculated joint trajectories. In distinction, for many movement tasks, animals and humans control limb impedance, allowing for a more robust handling of unexpected disturbances (Pratt 2002).

The development of animal-like and human-like robots that mimic the kinematics and kinetics of their biological counterparts, quantitatively or qualitatively, is indeed a formidable task. Humans, for example, are capable of performing numerous dynamical movements in a wide variety of complex and novel environments while robustly rejecting a large spectrum of disturbances. Given limitations on computational capacity, real-time trajectory planning in joint space does not seem feasible using optimization strategies with moderately-long future time horizons. Subsequently, for the diversity of biological motor tasks to be represented in a robot's movement repertoire, the control problem has to be restated using a lower dimensional representation (Full and Koditschek 1999). However, independent of the specific architecture that achieves that reduction in dimension, biomechanical motion characteristics have to be identified and appropriately addressed.

There are two ground reference points used for motion identification and control in biomechanics and legged robotics. The locations of these reference points relative to each other, and relative to the ground support area, provide important local and sometimes global characteristics of whole-body movement, serving as benchmarks for either physical or desired movement patterns. The Zero Moment Point (ZMP), first discussed by Elftman ${ }^{1}$ (1938) for the study of human biomechanics, has only more recently been used in the context of legged machine control (Vukobratovic and Juricic 1969; Vukobratovic and Stepanenko 1972; Takanishi et al. 1985; Yamaguchi, Takanishi and Kato 1993; Hirai 1997; Hirai et al. 1998). The Centroidal Moment Pivot (CMP) is yet another ground reference point recently introduced in the literature (Herr, Hofmann, and Popovic 2003; Hofmann, 2003; Popovic, Hofmann, and Herr 2004a; Goswami and Kallem 2004). When the CMP corresponds with the ZMP, the ground reaction force passes directly through the CM of the body, satisfying a zero moment or rotational equilibrium condition. Hence, the departure of the CMP from the ZMP is an indication of non-zero CM body moments, causing variations in whole-body, spin angular momentum.

In addition to these two standard reference points, Goswami (1999) introduced the Foot Rotation Indicator (FRI), a ground reference point that provides information on stance-foot angular accelerations when only one foot is on the ground. However, recent study (Popovic, Goswami and Herr 2005) find that the mean separation distance between the FRI and ZMP during the powered plantar flexion period of single support is within the accuracy of their

\footnotetext{
${ }^{1}$ Although Borelli (1680) discussed the concept of the ZMP for the case of static equilibrium, it was Elftman (1938) who introduced the point for the more general dynamic case. Elftman named the specified point the "position of the force" and built the first ground force plate for its measurement.
} 
measurement ( $0.1 \%$ of foot length), and thus the FRI point is determined not to be an adequate measure of foot rotational acceleration.

In this chapter we study the ZMP and CMP ground reference points. Using a consistent mathematical notation, we define and compare the ground points in Section 2.0 and outline the various methodologies that can be employed in their estimation. In Section 3.0, we analyze the ZMP and CMP trajectories for level-ground, steady-state human walking, and in Section 4.0, we conclude the paper with a discussion of the significance of the ground reference points to legged robotic control systems.

In Section 3.0, key hypothesis is tested regarding the nature of the ground reference points in level-ground, steady-state human walking. Because recent biomechanical investigations have shown that total spin angular momentum is highly regulated throughout the walking cycle (Popovic, Gu, and Herr 2002; Gu 2003; Herr, Whiteley and Childress 2003; Popovic, Hofmann, and Herr 2004a; Herr and Popovic 2004), we hypothesize that the CMP trajectory will never leave the ground support base throughout the entire walking gait cycle, closely tracking the ZMP trajectory throughout the single and double support phases of gait. We test both the CMP hypothesis using a morphologically realistic human model and kinetic and kinematic gait data measured from ten human subjects walking at self-selected walking speeds.

\section{ZMP and CMP Reference Points: Definitions and Comparisons}

In this section, we define the ground reference points: ZMP and CMP. Although the reference points have been defined previously in the literature, we define and compare them here using a consistent terminology and mathematical notation.

In this paper, we adopt a notation by which $\vec{\tau}\left(\vec{r}_{A}\right)$ symbolizes the total moment acting on a body about point $\vec{r}_{A}$. For example, $\vec{\tau}(0)$ symbolizes a moment calculated at the origin of a coordinate frame. This notation stresses the fact that a moment of force acting on a body changes depending on the point about which it is calculated. In addition to the point about which the moment is calculated, we also designate the force used in the moment calculation. For example, if we consider only the moment due to the ground reaction force acting on a body, we specify this with the subscript G.R., i.e. $\vec{\tau}_{G . R .}\left(\vec{r}_{A}\right)$. Also, in this paper when we consider only a moment that acts on a particular body segment, or group of segments, we specify that moment using the segment's name in the superscript, e.g. $\vec{\tau}^{\text {foot }}\left(\vec{r}_{A}\right)$. In addition, in this manuscript, we often refer to the ground support base (GSB) to describe the foot-ground interaction. The GSB is the actual foot-ground contact surface when only one foot is in contact with the ground, or the convex hull of the two or more discrete contact surfaces when two or more feet are in contact with the ground, respectively. Finally, the ground support envelope is used to denote the actual boundary of the foot when the entire foot is flat on the contact surface, or the actual boundary of the convex hull when two or more feet are flat on the contact surface. In contrast to the ground support base, the ground support envelope is not time varying even in the presence of foot rotational accelerations or rolling.

\subsection{Zero Moment Point (ZMP)}

In the book On the Movement of Animals, Borelli (1680) discussed a biomechanical point that he called the support point, a ground reference location where the resultant 
ground reaction force acts in the case of static equilibrium. Much later, Elftman (1938) defined a more general "position of the force" for both static and dynamic cases, and he built the first ground force plate for its measurement. Following this work, Vukobratovic and Juricic (1972) revisited Elftman's point and expanded its definition and applicability to legged machine control. They defined how the point can be computed from legged system state and mass distribution, allowing a robotic control system to anticipate future ground-foot interactions from desired body kinematics. For the application of robotic control, they renamed Elftman's point the Zero Moment Point (ZMP).

Although for flat horizontal ground surfaces the ZMP is equal to the center of pressure, the points are distinct for irregular ground surfaces. In the Appendix of this manuscript, we properly define these ground points, and prove their equivalence for horizontal ground surfaces, and their uniqueness for more complex contact topologies.

Vukobratovic and Juricic (1969) defined the ZMP as the "point of resulting reaction forces at the contact surface between the extremity and the ground". The ZMP, $\vec{r}_{Z M P}$, therefore may be defined as the point on the ground surface about which the horizontal component of the moment of ground reaction force is zero (Arakawa and Fukuda 1997; Vukobratovic and Borovac 2004), or

$$
\left.\vec{\tau}_{G . R .}\left(\vec{r}_{Z M P}\right)\right|_{\text {horizontal }}=0
$$

Equation (1) means that the resulting moment of force exerted from the ground on the body about the ZMP is always vertical, or parallel to $\vec{g}$. The ZMP may also be defined as the point on the ground at which the net moment due to inertial and gravitational forces has no component along the horizontal axes (Hirai et al. 1998; Dasgupta and Nakamura 1999; Vukobratovic and Borovac 2004), or

$$
\left.\vec{\tau}_{\text {inertia }+ \text { gravity }}\left(\vec{r}_{Z M P}\right)\right|_{\text {horizontal }}=0 .
$$

Proof that these two definitions are in fact equal may be found in Goswami (1999) and more recently in Sardain and Bessonet (2004).

Following from equation (1), if there are no external forces except the ground reaction force and gravity, the horizontal component of the moment that gravity creates about the ZMP is equal to the horizontal component of the total body moment about the ZMP,

$$
\begin{gathered}
\left.\vec{\tau}\left(\vec{r}_{Z M P}\right)\right|_{\text {horizontal }}, \text { or } \\
\left.\vec{\tau}\left(\vec{r}_{Z M P}\right)\right|_{\text {horizontal }}=\left[\left(\vec{r}_{C M}-\vec{r}_{Z M P}\right) \times M \vec{g}\right]_{\text {horizontal }}
\end{gathered}
$$

where $\vec{r}_{C M}$ is the center of mass (CM) and $M$ is the total body mass. Using detailed information of body segment dynamics, this can be rewritten as

$$
\sum_{i=1}^{N}\left[\left(\vec{r}_{i}-\vec{r}_{Z M P}\right) \times m_{i} \vec{a}_{i}+\frac{d\left(\vec{I}_{i} \vec{\omega}_{i}\right)}{d t}\right]_{\text {horizontal }}=\left[\left(\vec{r}_{C M}-\vec{r}_{Z M P}\right) \times M \vec{g}\right]_{\text {horizontal }}
$$

where $\vec{r}_{i}$ is the CM of the $i$-th link, $m_{i}$ is the mass of the $i$-th link, $\vec{a}_{i}$ is the linear acceleration of the $i$-th link CM, $\vec{I}_{i}$ is the inertia tensor of the $i$-th link about the link's CM, and $\vec{\omega}_{i}$ is the 
angular velocity of the $i$-th link. Equation (4) is a system of two equations with two unknowns, $x_{Z M P}$ and $y_{Z M P}$, that can be solved to give

$$
\begin{gathered}
x_{Z M P}=-\frac{\sum_{i=1}^{N}\left[\vec{r}_{i} \times m_{i}\left(\vec{a}_{i}-\vec{g}\right)+\frac{d\left(\vec{I}_{i} \vec{\omega}_{i}\right)}{d t}\right]_{Y}}{M\left(\ddot{Z}_{C M}+g\right)} \text { and } \\
y_{Z M P}=\frac{\sum_{i=1}^{N}\left[\vec{r}_{i} \times m_{i}\left(\vec{a}_{i}-\vec{g}\right)+\frac{d\left(\vec{I}_{i} \vec{\omega}_{i}\right)}{d t}\right]_{X}}{M\left(\ddot{Z}_{C M}+g\right)} .
\end{gathered}
$$

Given full body kinematics and the mass distribution of a legged system, equation (5) can be used to reconstruct the ZMP trajectory. Alternatively, at a particular instant in time, equation (5) can be employed as a constraint equation for deciding joint accelerations consistent with a desired ZMP position, as discussed by Kondak and Hommel (2003).

Finally, the ZMP as a function of the CM position, net CM force $\left(\vec{F}=M \vec{a}_{C M}\right)$, and net moment about the $\mathrm{CM}$ can be expressed as

$$
\begin{gathered}
x_{Z M P}=x_{C M}-\frac{F_{x}}{F_{z}+M g} z_{C M}-\frac{\tau_{y}\left(\vec{r}_{C M}\right)}{F_{z}+M g} \text { and } \\
y_{Z M P}=y_{C M}-\frac{F_{y}}{F_{z}+M g} z_{C M}+\frac{\tau_{x}\left(\vec{r}_{C M}\right)}{F_{z}+M g} .
\end{gathered}
$$

As emphasized in Figure 1, the most important notion of the ZMP quantity, applicable for both single and multi-leg ground support phases, is that it resolves the ground reaction force distribution to a single point. However, one needs to be careful to use this point in an appropriate manner. Most notably, both the vertical component of moment and the CM work performed by the ground reaction force cannot be computed solely on the bases of the $\mathrm{ZMP}$ trajectory and the resulting ground reaction force vector. For example, the resultant horizontal ground reaction force could be zero while the vertical component of moment and/or the work performed by the ground reaction force could be nonzero. Consider a legged posture in which the following conditions are satisfied: 1) the ZMP is located just beneath the $\mathrm{CM} ; 2$ ) the horizontal ground reaction force field is tangent to a circle centered about the ZMP; and 3) the horizontal ground reaction force magnitude is a function of only radial distance. In this situation, shown in Figure 2, the net horizontal force is zero, but the net moment is nonzero. Another example is two particles of equal mass subject to two forces equal in magnitude but acting in opposite directions; while the net force is zero and the CM is at rest, the particles are moving and the work conducted by the external forces is nonzero. In other words, neither $\delta W_{G . R .}=\vec{F}_{G . R} \delta \vec{r}_{Z M P}$ nor $\delta W_{G . R .}=\vec{F}_{G . R}, \delta r_{C M}$ are permissible expressions for the work performed by the ground reaction force. 


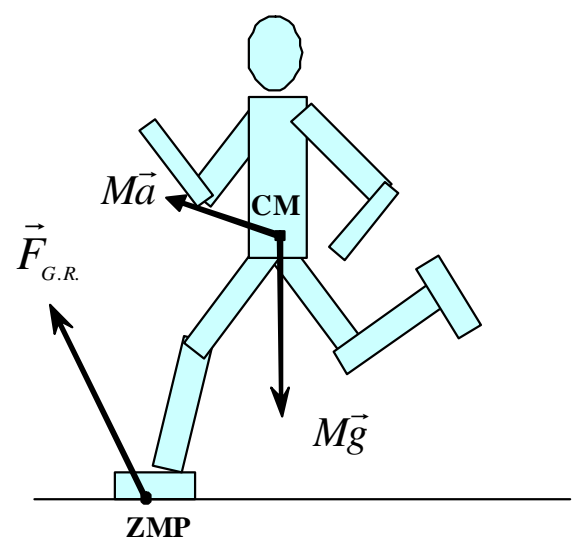

Fig. 1. Zero Moment Point (ZMP). The ZMP is where the ground reaction force acts whereas the $\mathrm{CM}$ point is where inertia and the force of gravity originate.

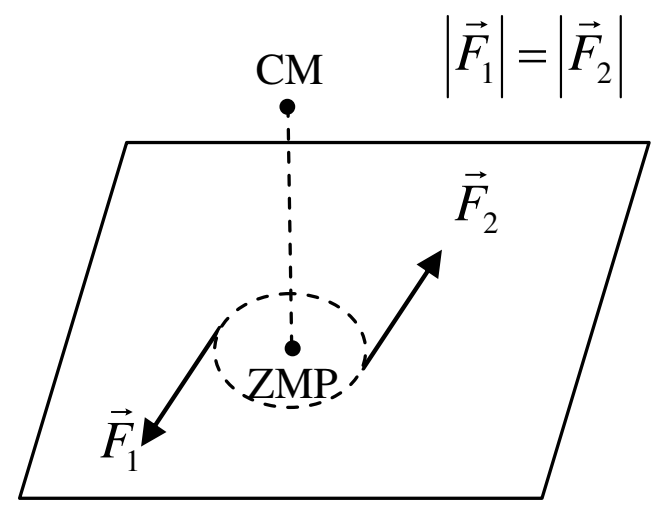

Fig. 2. A legged posture is shown in which the ZMP is located just beneath the CM, the horizontal ground reaction force field is tangent to a circle centered about the $\mathrm{ZMP}$, and the horizontal ground reaction force magnitude is a function of only radial distance. In this case, the net horizontal force is zero, but the net moment is nonzero. Thus, both the vertical component of moment and the CM work performed by the ground reaction force cannot be computed solely on the bases of the ZMP trajectory and the resulting ground reaction force vector.

\subsection{Centroidal Moment Pivot (CMP)}

\subsubsection{Motivation}

Biomechanical investigations have determined that for normal, level-ground human walking, spin angular momentum, or the body's angular momentum about the CM, remains small through the gait cycle. Researchers discovered that spin angular momentum about all three spatial axes was highly regulated throughout the entire walking cycle, including both single and double support phases, by observing small moments about the 
body's CM (Popovic, Gu and Herr 2002) and small spin angular momenta (Popovic, Hofmann, and Herr 2004a; Herr and Popovic 2004). In the latter investigations on spin angular momentum, a morphologically realistic human model and kinematic gait data were used to estimate spin angular momentum at self-selected walking speeds. Walking spin values were then normalized by dividing by body mass, total body height, and walking speed. The resulting dimensionless spin was surprisingly small. Throughout the gait cycle, none of the three spatial components ever exceeded 0.02 dimensionless units ${ }^{2}$.

To determine the effect of the small, but non-zero angular momentum components on whole body angular excursions in human walking, the whole body angular velocity vector can be computed, or

$$
\vec{\omega}(t)=\vec{I}^{-1}\left(\vec{r}_{C M}, t\right) \vec{L}\left(\vec{r}_{C M}, t\right) .
$$

Here the time dependent quantity, $\vec{I}\left(\vec{r}_{C M}, t\right)=\sum_{i=1}^{N} \vec{I}_{i}\left(\vec{r}_{C M}, t\right)$, is the whole body inertia tensor about the CM. Subsequently, the whole body angular velocity vector may be integrated to give the whole body angular excursion vector, or

$$
\vec{\theta}(t)=\int_{-\infty}^{t} \vec{\omega}\left(t^{*}\right) d t^{*}+C
$$

where $\mathrm{C}$ is an integration constant determined through an analysis of boundary conditions ${ }^{3}$ (Popovic, Hofmann, and Herr 2004a). The whole body angular excursion vector can be accurately viewed as the rotational analog of the $\mathrm{CM}$ position vector (i.e. note that analogously $\vec{v}=\dot{\vec{r}}_{C M}=M^{-1} \vec{p}$ and $\left.\vec{r}_{C M}(t)=\int_{-\infty}^{t} \vec{v}_{C M}\left(t^{*}\right) d t^{*}+D\right)$. In recent biomechanical investigations, angular excursion analyses for level ground human walking showed that the maximum whole body angular deviations within sagittal $\left(<1^{\circ}\right)$, coronal $\left(<0.2^{\circ}\right)$, and transverse $\left(<2^{\circ}\right)$ planes were negligibly small throughout the walking gait cycle (Popovic, Hofmann and Herr 2004a; Herr and Popovic 2005). These results support the hypothesis that spin angular momentum in human walking is highly regulated by the central nervous system (CNS) so as to keep whole body angular excursions at a minimum.

According to Newton's laws of motion, a constant spin angular momentum requires that the moments about the $\mathrm{CM}$ sum to zero. During the flight phase of running or

\footnotetext{
2Using kinematic data from digitized films (Braune and Fisher 1895), Elftman (1939) estimated spin angular momentum during the single support phase of walking for one human test subject, and found that arm movements during walking decreased the rotation of the body about the vertical axis. Although Elftman did not discuss the overall magnitude of whole body angular momentum, he observed important body mechanisms for intersegment cancellations of angular momentum.

3Since the whole body angular excursion vector defined in equation $(7 \mathrm{~b})$ necessitates a numerical integration of the body's angular velocity vector, its accurate estimate requires a small integration time span and a correspondingly small error in the angular velocity vector.
} 
jumping, angular momentum is perfectly conserved since the dominant external force is gravity acting at the body's CM. However, during the stance period, angular momentum is not necessarily constant because the legs can exert forces on the ground tending to accelerate the system (Hinrichs, Cavanagh and Williams 1983; Raibert, 1986; Dapena and McDonald 1989; LeBlanc and Dapena 1996; Gu 2003). Hence, a legged control system must continually modulate moments about the CM to control spin angular momentum and whole body angular excursions. For example, the moment about the CM has to be continually adjusted throughout a walking gait cycle to keep spin angular momentum and whole body angular excursions from becoming appreciably large. To address spin angular momentum and the moment about the CM in connection with various postural balance strategies, the CMP ground reference point was recently introduced (Herr, Hofmann, and Popovic 2003; Hofmann 2003; Popovic, Hofmann, and Herr 2004a). Goswami and Kallem (2004) proposed the same point in an independent investigation ${ }^{4}$.

\subsubsection{Definition}

The Centroidal Moment Pivot (CMP) is defined as the point where a line parallel to the ground reaction force, passing through the $\mathrm{CM}$, intersects with the external contact surface (see Figure 3). This condition can be expressed mathematically by requiring that the cross product of the CMP-CM position vector and the ground reaction force vector vanishes, or

$$
\left(\vec{r}_{C M P}-\vec{r}_{C M}\right) \times \vec{F}_{G . R .}=0 \text { and } z_{C M P}=0 .
$$

By expanding the cross product of equation (8), the CMP location can be written in terms of the $\mathrm{CM}$ location and the ground reaction force, or

$$
\begin{gathered}
x_{C M P}=x_{C M}-\frac{F_{G . R . X}}{F_{G . R . Z}} z_{C M} \text { and } \\
y_{C M P}=y_{C M}-\frac{F_{G . R . Y}}{F_{G . R . Z}} z_{C M} .
\end{gathered}
$$

Finally, by combining ZMP equation (6) and CMP equation (9), the CMP location may also be expressed in terms of the ZMP location, the vertical ground reaction force, and the moment about the CM, or

$$
\begin{gathered}
x_{C M P}=x_{Z M P}+\frac{\tau_{y}\left(\vec{r}_{C M}\right)}{F_{G . R . Z}} \text { and } \\
y_{C M P}=y_{Z M P}-\frac{\tau_{x}\left(\vec{r}_{C M}\right)}{F_{G . R . Z}} .
\end{gathered}
$$

4Popovic, Hofmann, and Herr (2004a) named the specified quantity the Zero Spin Center of Pressure (ZSCP) point, whereas Goswami and Kallem (2004) named the specified quantity the Zero Rate of Angular Momentum (ZRAM) point. In this manuscript, a more succinct name is used, or the Centroidal Moment Pivot (CMP) (Popovic, Goswami, and Herr 2005). 


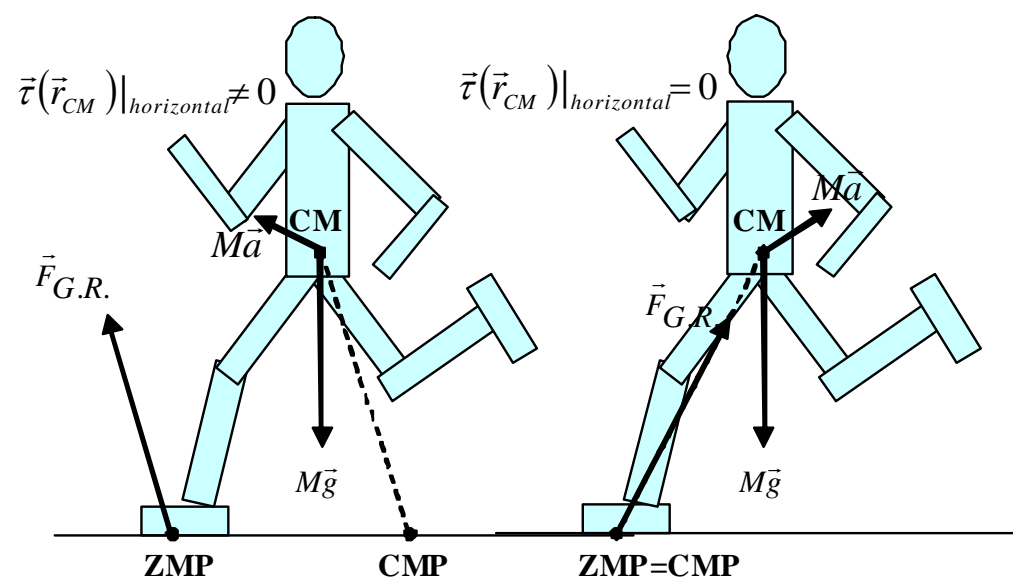

Fig. 3. Centroidal Moment Pivot (CMP). The CMP is the point where the ground reaction force would have to act to keep the horizontal component of the whole body angular momentum constant. When the moment about the CM is zero (shown in the figure to the right), the CMP coincides with the ZMP. However, when the CM moment is non-zero (figure on the left), the extent of separation between the CMP and ZMP is equal to the magnitude of the horizontal component of moment about the $\mathrm{CM}$, divided by the normal component of the ground reaction force.

As is shown by equation (10), when the CMP is equal to the $\mathrm{ZMP}$, the ground reaction force passes directly through the $\mathrm{CM}$ of the body, satisfying a zero moment or rotational equilibrium condition. In distinction, when the CMP departs from the ZMP, there exists a non-zero body moment about the $\mathrm{CM}$, causing variations in whole-body, spin angular momentum. While by definition the ZMP cannot leave the ground support base, the CMP can -- but only in the presence of a significant moment about the CM. Hence, the notion of the CMP, applicable for both single and multi-leg ground support phases, is that it communicates information about whole body rotational dynamics when supplemented with the ZMP location (excluding body rotations about the vertical axis).

It is interesting to note that when the stance foot is at rest during single support, and when there is zero moment about the $\mathrm{CM}$, the ZMP and CMP coincide. However, generally speaking, these ground reference points cannot be considered equivalent.

\section{The ZMP, FRI and CMP trajectories in human walking}

For the diversity of biological motor tasks to be represented in a robot's movement capabilities, biomechanical movement strategies must first be identified, and legged control systems must exploit these strategies. To this end, we ask what are the characteristics of the ZMP and CMP ground reference points in human walking, and how do they interrelate? As discussed in Section 2.0, spin angular momentum remains small throughout the walking cycle. Hence, we hypothesize that the CMP trajectory will never leave the ground support base during the entire walking gait cycle, closely tracking the ZMP trajectory during both single and double support phases. 
In this section we test the CMP hypothesis using a morphologically realistic human model and kinetic and kinematic gait data measured from ten human subjects walking at selfselected forward walking speeds. In Section 3.1, we outline the experimental methods used in the study, including a description of data collection methods, human model structure and the analysis procedures used to estimate, compare and characterize the reference point biological trajectories. Finally, in Section 3.2, we present the experimental results of the gait study, and in Section 3.3, we discuss their significance.

\subsection{Experimental Methods}

\subsubsection{Kinetic and Kinematic Gait Measures}

For the human walking trials, kinetic and kinematic data were collected in the Gait Laboratory of Spaulding Rehabilitation Hospital, Harvard Medical School, Boston, MA, in a study approved by the Spaulding Committee on the Use of Humans as Experimental Subjects. Ten healthy adult participants, five male and five female, with an age range from 20 to 38 years old, were involved in the study.

Participants walked at a self-selected forward speed over a 10-meter long walkway. To ensure a consistent walking speed between experimental trials, participants were timed across the 10-meter walking distance. Walking trials with forward walking speeds within a $\pm 5 \%$ interval were accepted. Seven walking trials were collected for each participant.

To assess gait kinematics, an eight infrared-camera, motion analysis system (VICON 512 System, Oxford Metrics, Oxford, England) was used to measure the three-dimensional positions of reflective markers placed on various parts of each participant's body. The frame rate of the camera system was 120 frames per second. A total of 33 markers were employed: sixteen lower extremity markers, five thoracic and pelvic markers, eight upper extremity markers, and four head markers. The markers were attached to the following bony landmarks: bilateral anterior superior iliac spines, posterior superior iliac spines, lateral femoral condyles, lateral malleoli, forefeet and heels. Additional markers were rigidly attached to wands over the mid-femur and mid-shaft of the tibia. Kinematic data of the upper body were also collected with markers placed on the following locations: sternum, clavicle, C7 vertebra, T10 vertebra, head, and bilaterally on the shoulder, elbow and wrist. Depending on the position and movement of a participant, the system was able to detect marker position with a precision of a few millimeters.

During walking trials, ground reaction forces were measured synchronously with the kinematic data using two staggered force platforms (model OR6-5-1, AMTI, Newton, MA) embedded in the 10-meter walkway. The force data were collected at a sampling rate of $1080 \mathrm{~Hz}$ at an absolute precision of $\sim 0.1 \mathrm{~N}$ for ground reaction forces and $\sim 1 \mathrm{~mm}$ for the ZMP location.

\subsubsection{Human Model Structure}

A morphologically realistic human model was constructed in order to calculate the FRI and CMP ground reference trajectories. The human model, shown in Figure 4, consisted of 18 links: right and left forefoot links, heels, shanks, thighs, hands, forearms, upper arms, pelvis-abdomen region, thorax, neck and head. The forefoot and a heel sections, as well as the hands, were modeled as rectangular boxes. The shanks, thighs, forearms and upper arms were modeled as truncated cones. The pelvis-abdomen region and the thoracic link were modeled as elliptical slabs. The neck was modeled as a cylinder, and the head was modeled as a sphere. 

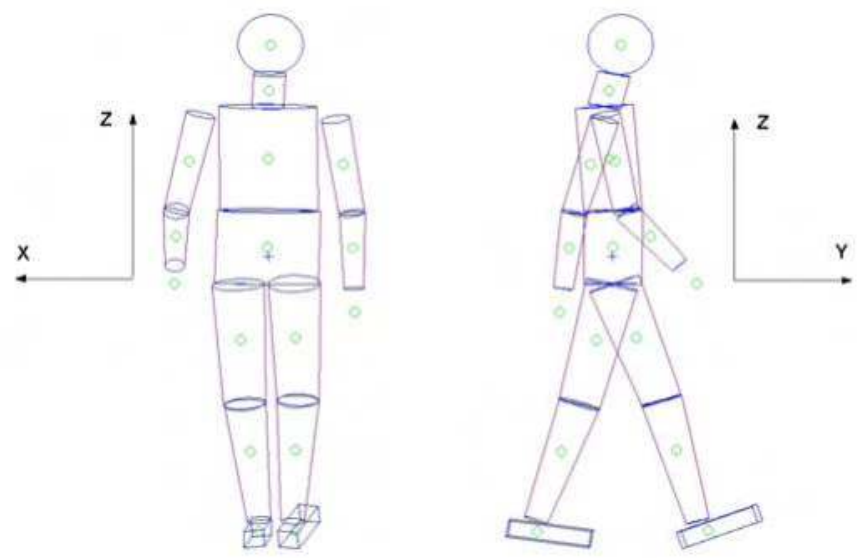

Fig. 4. The morphologically realistic human model used in the human gait study. The human model has a total of 38 degrees of freedom, or 32 internal degrees of freedom (12 for the legs, 14 for the arms and 6 for the rest) and 6 external degrees of freedom (three body translations and three rotations). Using morphological data from the literature and direct human participant measurements, mass is distributed throughout the model's links in a realistic manner.

To increase the accuracy of the human model, twenty-five length measurements were taken on each participant: 1) foot and hand length, width and thickness; 2) shanks, thighs, forearms and upper arm lengths as well as their proximal and distal base radii; 3 ) thorax and pelvis-abdomen heights, widths and thicknesses; and 4) radius of the head. The neck radius was set equal to half the head radius.

Using observations of the human foot's articulated bone structure (Ankrah and Mills 2003), the mass of the forefoot was estimated to be $20 \%$ of the total foot mass. For the remaining model segments, a link's mass and density were optimized to closely match experimental values in the literature (Winter 1990; Tilley and Dreyfuss 1993) using the following procedure. The relative mass distribution throughout the model, described by a 16component vector $D$, (i.e. the heel and forefoot were represented as a single foot segment) was modeled as a function of a single parameter $\alpha$ such that

$$
D(\alpha)=\left(D_{A}+\alpha D_{S}\right) /(1+\alpha) .
$$

Here $D_{A}$ is the average relative mass distribution obtained from the literature (Winter 1990), and the subject specific relative mass distribution, $D_{S}$, was obtained by using an equal density assumption; the relative mass of the i-th link, $D_{S}{ }^{i}$, was assumed to be equal to the ratio of the link's volume, $V^{i}$, over the total body volume, $V$, or $D_{S}{ }^{i}=V^{i} / V$. The selection of parameter $\alpha$ then uniquely defined the density profile throughout the various links of the human model, as described by the 16-component vector $P(\alpha)$, such that 
$P^{i}(\alpha)=M D^{i}(\alpha) / V^{i}$ where $M$ was equal to the total body mass. The resulting relative mass distribution, $D_{R}$, was obtained as $D_{R}=D\left(\alpha_{\min }\right)$ where $\alpha_{\min }$ minimized the absolute error between the distribution of link densities, $P(\alpha)$, and the average distribution of link densities obtained from the literature, $P_{A}$ (Winter 1990). In notation form, this analysis procedure may be expressed as

$$
\min \left|P(\alpha)-P_{A}\right|=\min \sqrt{\sum_{i}\left[P^{i}(\alpha)-P_{A}^{i}\right]^{2}} \Rightarrow \alpha_{\min } \Rightarrow D_{R}=\frac{D_{A}+\alpha_{\min } D_{S}}{1+\alpha_{\min }} .
$$

\subsubsection{Data Analysis}

For each participant and for each walking trial, the ZMP and CMP trajectories were computed. The ZMP was estimated directly from the force platform data using equation (1). The CMP was calculated using the calculated CM position from the human model, and the measured ZMP and ground reaction force data from the force platforms (see equation (9)). Here the $\mathrm{CM}$ trajectory was estimated by computing the $\mathrm{CM}$ of the human model at each gait posture throughout the entire gait cycle. The model's posture, or spatial orientation, was determined from the joint position data collected from the human gait trials.

As a measure of how well well the CMP tracked the ZMP, we computed the linear distance between the CMP and the ZMP, at each moment throughout the gait cycle. For each participant, the mean CMP-ZMP distance was then computed using all seven gait trials. This mean distance was then normalized by the participant's foot length. We then performed a nonparametric Wilcoxon signed rank test for zero median to test for significance in the mean CMP-ZMP distance between the single and double support phases of gait ( $\mathrm{N}=10$ subjects). For this statistical analysis, significance was determined using $\mathrm{p}<$ 0.05 .

\subsection{Results}

Representative trajectories of the ZMP and CMP are shown in Figure 5 for a healthy female participant (age 21, mass $50.1 \mathrm{~kg}$, height $158 \mathrm{~cm}$, speed $\sim 1.3 \mathrm{~m} / \mathrm{s}$ ). For each study participant, Table 1 lists the mean normalized distances between the CMP and the ZMP.

For all participants and for all walking trials, the ZMP was always well inside the ground support base. The ZMP was never closer to the edge of the ground support base than by approximately $5-10 \%$ of foot length (see Figure 5). Finally, for all participants and for all walking trials, the CMP remained within ground support base throughout the entire gait cycle. The mean of the normalized distance between the CMP and the ZMP for the single support phase $(14 \pm 2 \%)$ was not significantly different from that computed for the double support phase $(13 \pm 2 \%)(p=0.35)$.

\subsection{Discussion}

Since spin angular momentum has been shown to remain small throughout the walking cycle, we hypothesize that the CMP will never leave the ground support base throughout the entire gait cycle, closely tracking the ZMP. The results of this investigation support this hypothesis. We find that the CMP never leaves the ground support base, and the mean separation distance between the CMP and ZMP is small (14\% of foot length), highlighting how closely the human body regulates spin angular momentum in level ground walking. 
The mean normalized distance between the CMP and the ZMP for the single support phase $(14 \pm 2 \%)$ was not significantly different from that computed for the double support phase $(13 \pm 2 \%) \quad(p=0.35)$, suggesting that the CMP is a reasonable estimate of ZMP position independent of the number of legs in contact with the ground surface.

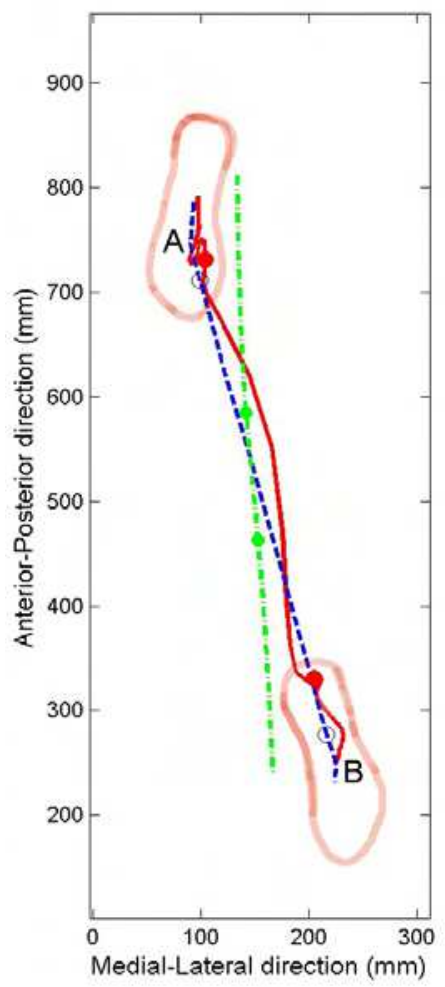

Fig. 5. Plotted are the ZMP (dashed), CMP (solid) and CM ground projection (dasheddotted) trajectories and corresponding footprints of a study participant walking at a selfselected speed $(1.3 \mathrm{~m} / \mathrm{s})$. The two circles on each line denote the transition from single to double support and vice versa. Data span from the middle of a single support phase to the middle of the next single support phase of the opposite limb.

\begin{tabular}{|c|c|c|c|c|c|c|c|c|c|c|c|}
\hline Subjects & $\mathbf{1}$ & $\mathbf{2}$ & $\mathbf{3}$ & $\mathbf{4}$ & $\mathbf{5}$ & $\mathbf{6}$ & $\mathbf{7}$ & $\mathbf{8}$ & $\mathbf{9}$ & $\mathbf{1 0}$ & Mean \pm STD \\
\hline $\boldsymbol{A} \%$ & 16 & 14 & 13 & 17 & 16 & 10 & 12 & 11 & 15 & 15 & $\mathbf{1 4} \pm \mathbf{2}$ \\
\hline $\boldsymbol{B} \%$ & 15 & 13 & 10 & 15 & 12 & 9.0 & 14 & 15 & 15 & 14 & $\mathbf{1 3} \pm \mathbf{2}$ \\
\hline $\boldsymbol{C} \%$ & 16 & 13 & 12 & 16 & 15 & 10 & 12 & 12 & 15 & 15 & $\mathbf{1 4} \pm \mathbf{2}$ \\
\hline
\end{tabular}

Table 1. For ten healthy test participants walking steadily at their self-selected speeds, listed are the mean distances, normalized by foot length, between the CMP and the ZMP points for the single support phase (A), double support phase (B), and across the entire gait cycle (C). 


\section{Control Implications of Ground Reference Points ZMP and CMP}

In this section, we discuss how the ZMP and CMP ground reference points can be used in legged robotic and prosthetic control systems. In Section 4.1, the control implications of the foot-ground ZMP are discussed. In Section 4.2, we discuss how the control of both the ZMP and the CMP could enhance postural stability for single-leg standing.

\subsection{Control Implications of the ZMP}

\subsubsection{Does a ZMP Location Inside the Ground Support Base Indicate Postural Stability?}

As noted by Goswami (1999), the requirement that the ZMP should be inside the ground support base has been extensively used in the literature as a criterion of postural stability 5 (Shih et al. 1990; Li, Takanishi and Kato 1993; Shih 1996; Arakawa and Fukuda 1997; Huang et al. 2001). However, since the ZMP must always reside within the ground support base as required by fundamental physics (see equation (1)), a ZMP estimate that falls outside the ground support base should be an indication of non-physical behavior and not an indication of overall postural instability. For example, if a computer simulation predicts that the ZMP is outside the ground support base, the result should simply be viewed as a non-physical simulation artifact and not an indication of postural instability. Still further, if the simulation predicts a ZMP location within the ground support base, overall postural stability is not, in any way, guaranteed.

\subsubsection{Does Maintaining the ZMP at the Center of the Ground Support Envelope Guarantee Postural Stability?}

It has been suggested in the literature that postural stability during single support will be ensured if the ZMP remains at the center of the ground support envelope (Vukobratovic and Juricic 1969; Vukobratovic and Stepanenko 1973; Li, Takanishi and Kato 1993; Arakawa and Fukuda 1997; Huang et al. 2001). However, it is noted here that accurately controlling the ZMP location to coincide with the center of the ground support envelope will not in itself guarantee postural stability for all legged control problems. To clarify this point, consider the simple model of single support standing shown in Figure 6A. The mass of the body is represented as a point mass attached to a massless foot and leg linkage, and the ankle is the only actuated degree of freedom.

If the ZMP is tightly controlled to operate at the center of the ground support envelope, such that $x_{Z M P}=0$, then according to equation (6)

$$
F_{x}=M \ddot{x}_{C M}=M\left(\ddot{z}_{C M}+g\right) \frac{x_{C M}}{z_{C M}}-\frac{\tau_{y}}{z_{C M}} .
$$

For this simplified model, the moment about the CM is always equal to zero, $\tau_{y}=0$, since the mass of the body is represented as a single point mass. Thus, from equation (13) we have

5 Throughout this manuscript, postural stability, or body stability, refers to the maintenance of body attitude angles within a specified bounded region and the return to that bounded region after a perturbation (Vukobratovic, Frank and Juricic 1970). 


$$
\ddot{x}_{C M}=\left(\ddot{z}_{C M}+g\right) \frac{x_{C M}}{z_{C M}} .
$$

We see from equation (14) that for this simplified model, a control system that maintains the ZMP position at the center of the ground support envelope, or $x_{Z M P}=0$, causes the system to be equivalent to a statically unstable, non-actuated inverted pendulum. Thus, we may conclude that controlling the ZMP to operate at the center of the ground support envelope during single support cannot, by itself, ensure postural stability.
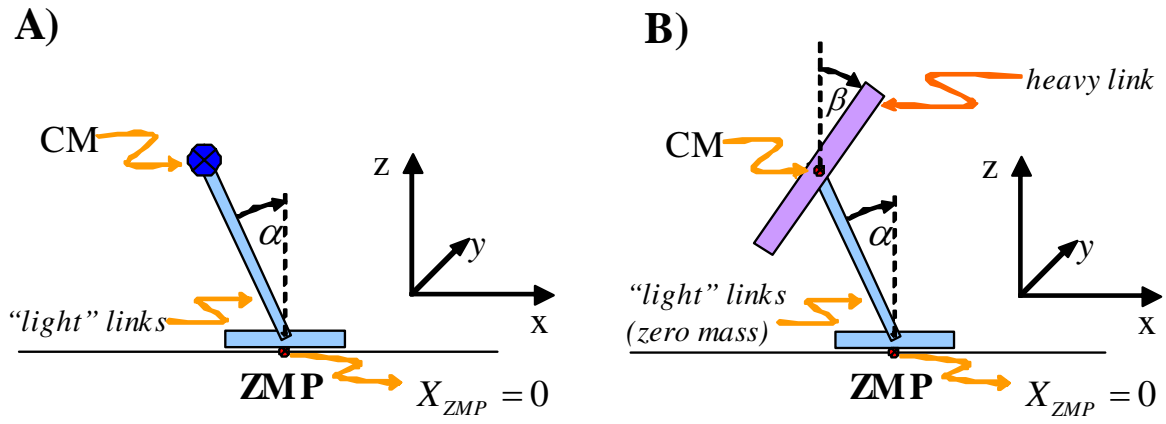

Fig. 6. In (A), a simple model of single-leg standing is shown consisting of three links: 1) a body link represented by a point mass equal to total body mass; 2) a massless leg link representing the stance leg; and 3) a massless foot link (base of support), which is aligned with the ground and which has limited extent. The ankle joint between the foot link and the leg link is the only actuated degree of freedom in the model. In (B), the same model as in (A) is shown except the body link is modeled as a solid uniform rod. In contrast to the model of (A), the model of (B) has an actuated ankle and hip joint. Thus, this model may have a nonzero moment about its CM.

If we now allow for non-zero ZMP positions, we obtain

$$
\ddot{x}_{C M}=\left(\ddot{z}_{C M}+g\right) \frac{x_{C M}-x_{Z M P}}{z_{C M}} .
$$

Thus, we see from equation (15) that by selecting an appropriate non-zero ZMP trajectory, the model of Figure 6A can be stabilized albeit for relatively modest CM disturbances. ${ }^{6}$ For example, if the $\mathrm{CM}$ projection onto the ground extends beyond the boundaries of the foot as a result of a disturbance to the system, the system cannot be stabilized simply by controlling ZMP position because the foot is not physically attached to the ground surface (see equation (15)) (Popovic and Herr 2003; Hofmann et al. 2004, Popovic, Hofmann and Herr 2004b).

\footnotetext{
${ }^{6}$ Here stability refers to the capacity of the system to restore the $\mathrm{CM}$ to a location vertically above the center of the ground support envelope $\left(x_{Z M P}=0\right)$ after a perturbation.
} 
Although controlling ZMP position is one strategy for stabilizing legged posture, it is not the only tool for addressing stability. For example, during single-leg standing, consider shrinking the stance foot to a single point. The ZMP is then constrained at that contact point and cannot be repositioned using a ZMP control strategy. As is apparent from equation (13), the only way to stabilize such a system is to produce a non-zero moment about the CM. In Section 4.2, we argue that by controlling both the ZMP and CMP ground reference positions, overall postural stability during single support standing can be maintained even in the presence of large disturbances where the CM projection on the ground surface extends beyond the ground support envelope.

\subsection{Control Implications of the CMP}

4.2.1 For Whole Body Rotational Control, Should a Control System Minimize CM Moment, Spin Angular Momentum, or Whole-Body Angular Excursions?

As noted in Section 3.2, the CMP trajectory was confined to the ground support base for each subject and for each walking trial. Thus, one metric of human-like walking that may be useful in the evaluation of biomimetic humanoid robots is that the CMP must remain within the ground support base, near the $\mathrm{ZMP}$, throughout the entire gait cycle. However, a zero moment about the CM, or a zero CMP-ZMP separation, should only be viewed as a condition of body rotational equilibrium and not a condition of postural stability. A loss of rotational equilibrium does not necessarily mean that the person or robot is destined to fall. In fact, the moment about the CM is prominently non-zero for many stable legged movement patterns (Hinrichs, Cavanagh and Williams 1983; Dapena and McDonald 1989; LeBlanc and Dapena 1996; Gu 2003). Non-zero CM moments are expected since the application of CM moment by a legged control system can increase the restoring force applied to the CM, as shown by equation (6), restoring $\mathrm{CM}$ position to a desired location (Popovic, Hofmann, and Herr 2004a, b; Hofmann et al. 2004).

Since the application of moments about the CM is one critical control strategy to achieve postural stability in the presence of disturbances, the objective for the controller of wholebody angular behavior should not be to achieve a zero $\mathrm{CM}$ moment, or equivalently, a zero CMP-ZMP separation. Rather, a CM moment should be applied by the system controller to achieve a desired spin angular momentum and a particular whole-body angular excursion (see equation (7)). For example, focusing solely on rotational degrees of freedom, one could write a simple $2^{\text {nd }}$ order differential control equation for a desired target moment, or

$$
\vec{\tau}_{\text {des. }}\left(\vec{r}_{C M}\right)=\dot{\vec{L}}_{\text {des. }}+\vec{a} \Delta \vec{\theta}+\vec{b} \Delta \dot{\vec{\theta}}=\dot{\vec{L}}_{\text {des. }}+\vec{a} \Delta \vec{\theta}+\vec{b}^{\prime} \Delta \vec{L}\left(\vec{r}_{C M}\right),
$$

where $\Delta \vec{L}\left(\vec{r}_{C M}\right)=\vec{L}\left(\vec{r}_{C M}\right)-\vec{L}_{\text {des. }}\left(\vec{r}_{C M}\right)$ and $\Delta \vec{\theta}=\vec{\theta}-\vec{\theta}_{\text {des. }}$, $\vec{a}$ and $\vec{b}$ (with $\vec{b}^{\prime}=\vec{b} \vec{I}^{-1}\left(\vec{r}_{C M}\right)$ ) are second order tensors, i.e. $3 \times 3$ matrices representing rotational "stiffness" and "damping" coefficients respectively, $\vec{I}\left(\vec{r}_{C M}\right)=\sum_{i=1}^{N} \vec{I}_{i}\left(\vec{r}_{C M}\right)$ is the whole body moment of inertia tensor about the $C M$ (also a function of time) and $\vec{\omega}=\vec{I}^{-1}\left(\vec{r}_{C M}\right) \vec{L}\left(\vec{r}_{C M}\right)$ is the whole body effective angular velocity (that may be integrated to give $\vec{\theta}$ ), see equation (7). Alternatively, instead of whole 
body angular excursions, which are not directly measurable quantities, one may consider using whole body principal angles defined by the relative orientations of the principal axes of the whole body moment of inertia tensor with respect to the non-rotating lab frame axes (Popovic and Herr 2005). For a humanoid walking robot, the desired whole body angular excursion and the spin angular momentum would both be set to zero and the rotational stiffness and damping coefficients would then be adjusted to achieve a desired system response.

In his book Legged Robots that Balance, Raibert (1986) speculated that a control system that keeps angular momentum constant during stance could achieve higher efficiency and better performance. Motivated by biomechanical measurements showing the relatively small size of CM moments during human walking, Popovic, Gu and Herr (2002) suggested that humanoid control systems should explicitly minimize global spin angular momentum during steady state forward walking $\left(\vec{L}_{\text {des. }}\left(\vec{r}_{C M}\right)=0\right)$. Using this approach, the zero-spin controller would apply corrective moments to minimize body spin when the whole body state is such that spin is non-zero. It is noted here that a consequence of this control objective is that the CMP-ZMP separation distance is minimized. However, a control system that only minimizes the CMP-ZMP separation distance will only ensure a constant spin angular momentum and not specifically a zero spin value.

Kajita et al. $(2003$; 2004) implemented a zero-spin control on the humanoid robot HRP-2 and showed its usefulness in kicking, hopping and running. Still further, Popovic, Hofmann and Herr (2004a) showed in a 2-D numerical simulation of walking that biologically realistic leg joint kinematics emerge through the minimization of spin angular momentum and the total sum of joint torque squared (minimal effort criteria), suggesting that both angular momentum and energetic factors may be important considerations for biomimetic controllers.

\subsubsection{Would Controlling Both ZMP and CMP Enhance Postural Stability?}

For the simplified model of single-leg standing shown in Figure 6A, Section 4.1, ankle torques have to be applied to move the ZMP such that appropriately needed horizontal forces are generated, as dictated by equation (15), to move the model's CM back over the foot support envelope. However, as required by physics (see equation (1)), the ZMP cannot leave the ground support base. This physical constraint poses a restriction on the magnitude of the restoring $\mathrm{CM}$ forces that can be applied by the system controller to restore $\mathrm{CM}$ position, and therefore, directly limits the range of perturbation that can be rejected by the system.

Let us now relax the zero moment condition $(\mathrm{CMP}=\mathrm{ZMP})$ and consider the model shown in Figure 6B. In that model, the point mass of model 6A is replaced with a uniform rod that rotates about a hip joint at the top of a massless leg and foot linkage. By controlling both the $\mathrm{ZMP}$ and CMP trajectories, a larger set of perturbations can be rejected than when controlling only the ZMP trajectory (Popovic, Hofmann and Herr 2004a,b; Hofmann et al. 2004). Even when the $\mathrm{ZMP}$ is at the very edge of the ground support envelope in the model of Figure $6 \mathrm{~B}$, a horizontal restoring force can still be produced through the application of a moment about the $\mathrm{CM}$, or equivalently by controlling the CMP relative to the ZMP. According to equation (6), the horizontal restoring force output of the model shown in Figure $6 \mathrm{~B}$ can now be written as

$$
F_{x}=M \ddot{x}_{C M}=M\left(\ddot{z}_{C M}+g\right) \frac{x_{C M}-x_{Z M P}}{z_{C M}}-\frac{\tau_{y}}{z_{C M}}=F_{x}^{\text {zero-moment }}+F_{x}^{\text {moment }}
$$


where $F_{x}^{\text {zero-moment }}=M\left(\ddot{z}_{C M}+g\right) \frac{x_{C M}-x_{Z M P}}{z_{C M}}$ corresponds to a Zero-Moment Balance Strategy and $F_{x}^{\text {moment }}=-\frac{\tau_{y}}{z_{C M}}$ corresponds to a Moment Balance Strategy. Because the CMP represents a unique pivot point, equation (17) may be written more compactly as

$$
F_{x}=M\left(\ddot{z}_{C M}+g\right) \frac{x_{C M}-x_{C M P}}{z_{C M}} .
$$

As highlighted by equation (18), the $\mathrm{CM}$ restoring force can be controlled by modulating the separation distance between the CM projection on the ground surface and the CMP location. Depending on the character of a particular movement task and robotic structure, the two balance control strategies may have different levels of influence on postural stability. For example, in Figure 6B, if the model's foot link were made infinitely small, with $x_{Z M P}=0$ as a physical constraint, the Moment Balance Strategy $(\mathrm{CMP} \neq \mathrm{ZMP})$ would necessarily dominate. However, when the CMP is in the vicinity of the ground support envelope boundary during single-leg balancing, or outside that boundary, the Moment Balance Strategy (CMP $\neq \mathrm{ZMP}$ ) must dominate since ZMP trajectory control alone cannot restore postural balance (Popovic, Hofmann and Herr 2004a,b; Hofmann et al. 2004). Therefore, the CMP location relative to the ground support envelope is an important indicator for a control system to determine which balance strategy should necessarily dominate (Popovic, Hofmann and Herr 2004a,b; Hofmann et al. 2004).

\section{Summary}

For the diversity of biological motor tasks to be represented in a robot's movement repertoire, biomechanical movement strategies must first be identified, and legged robotic control systems must exploit these strategies. To this end, in this chapter we ask what are the characteristics of the ZMP and CMP ground reference trajectories in human walking, and how do they interrelate? We compute walking reference trajectories using a human model and gait data measured from ten human subjects walking at self-selected speeds. We find that the CMP never leaves the ground support base, and the mean separation distance between the CMP and the ZMP is small (14\% of foot length) across both single and double support walking phases, highlighting how closely the human body regulates spin angular momentum in level ground walking.

We conclude the chapter with a discussion of legged control issues related to the ground reference points. Using a simple model of single-leg balancing, we show that by controlling both the ZMP and the CMP trajectories, larger CM restoring forces can be applied by a system than would be possible using only a ZMP control. An area of future research of considerable importance will be in the implementation of legged systems that control both the ZMP and the CMP locations, resulting in corrective $\mathrm{CM}$ forces and moments necessary to restore $\mathrm{CM}$ position and body angular orientation. Another area of future research will be to characterize the ZMP and CMP biological trajectories for a whole host of animal and human movement patterns in the hope to further motivate biomimetic control schemes. It is our hope that this work will lead to further studies in ground reference points for the identification and control of legged systems, resulting in an even wider range of locomotory performance capabilities of legged robots and prostheses. 


\section{Appendix: Center of Pressure (CP) and Zero Moment Point (ZMP): Equivalence and Uniqueness}

Although several authors (Goswami 1999; Sardain and Bessonet 2004) have speculated that the $\mathrm{CP}$ should be equivalent to the $\mathrm{ZMP}^{7}$, no formal proof has yet been advanced. In this Appendix, we put forth a formal proof of their equivalence for horizontal ground surfaces, and then we show their uniqueness for more complex contact topologies.

\section{The Equivalence of the ZMP and the CP for Horizontal Contact Surfaces}

The concept of CP most likely originated from the field of fluid dynamics. CP is utilized in aero-dynamical calculations of aircraft and rockets (Darling 2002). It is also frequently used in the study of human gait and postural balance (Winter 1990; Rose and Gamble 1994).

For a body resting on a flat horizontal ground surface, the position of the $\mathrm{CP}$, denoted by $\vec{r}_{C P}$, is defined as

$$
\vec{r}_{C P}=\frac{\int_{g s b} \vec{r} p(\vec{r}) d a}{\int_{g s b} p(\vec{r}) d a}=\frac{\left.\vec{\tau}_{G . R .}(0)\right|_{\text {horizontal }}}{F_{\text {G.R.Z }}} \times \frac{\vec{g}}{g},
$$

where the integration is over the ground support base (gsb), $d a$ is an infinitesimal element of the support surface located at $\vec{r}, p(\vec{r})$ is the pressure at that location, $F_{G . R . Z}$ is the vertical component of the resulting ground reaction force, and $\mathrm{g}$ is the gravitational acceleration. The second equality in equation (A.1) follows from $p(\vec{r}) d a=d F_{G . R . Z}$ and $\vec{r} \cdot \vec{g}=0$.

The resulting moment exerted from the ground on the body about the origin of the lab reference frame (assumed here to be on the ground) is

$$
\left.\vec{\tau}_{G . R .}(0)\right|_{\text {horizontal }}=\left.\int_{g s b}\left(\vec{r} \times d \vec{F}_{G . R .}\right)\right|_{\text {horizontal }}=-\int_{g s b}\left(\vec{r} \times \frac{\vec{g}}{g}\right) p(\vec{r}) d a=\frac{\vec{g}}{g} \times \int_{g s b} \vec{r} p(\vec{r}) d a .
$$

For simplicity we assume a horizontal ground surface in equations (A.1) and (A.2). However, the results may easily be generalized to include inclined surfaces as well if vector $-\vec{g} / g$ is exchanged for $\vec{n}_{\perp}$, the unit vector normal to the surface and pointing away from the ground. In addition, $F_{G . R . Z}$ has to be exchanged with $F_{G . R . \perp}$, the component of the ground reaction force normal to the surface, and $\left.\vec{\tau}\right|_{\text {horizontal }}$ has to be exchanged for $\left.\vec{\tau}\right|_{\|}$ where $\left.\vec{\tau}\right|_{\|} \cdot \vec{n}_{\perp}=0$. For a more complicated surface geometry, for example when two robot legs are posed on two surfaces of different inclination, the unique embedding surface does not exist. Below, we resolve this issue by considering an embedding convex volume instead of an embedding flat surface. The flat surface approach was first proposed by Takanishi et al. (1990) and later used by Sardain and Bessonnet (2004).

7Goswami (1999) and Sardain \& Bessonet (2004) did not prove the equivalence of the CP and the ZMP, but rather, they proved the equivalence of two definitions of the ZMP (see Section 2.1 for ZMP definitions, equations (1) and (2)). 
Given the definition of the $\mathrm{CP}$ (equation (A.1)), we can prove that the $\mathrm{CP}$ is identical to the $\mathrm{ZMP}$ by noting from equation (A.2) that

$$
\left.\vec{\tau}_{G . R .}\left(\vec{r}_{C P}\right)\right|_{\text {horizontal }}=\left.\vec{\tau}_{\text {G.R. }}(\mathbf{0})\right|_{\text {horizontal }}+F_{\text {G.R.Z }} \vec{r}_{C P} \times \frac{\vec{g}}{g}=\mathbf{0},
$$

therefore satisfying one definition of the ZMP defined in equation (1), Section 2.1.

Alternatively one could rewrite equation (1), Section 2.1 as,

$$
\left.\vec{\tau}_{G . R .}\left(\vec{r}_{Z M P}\right)\right|_{\text {horizontal }}=\int_{g s b}\left[\left(\vec{r}-\vec{r}_{Z M P}\right) \times d \vec{F}_{G . R .}\right]_{\text {horizontal }}=-\int_{g s b}\left[\left(\vec{r}-\vec{r}_{Z M P}\right) \times \frac{\vec{g}}{g}\right] d F_{G . R . Z}=0,
$$

to show that it is exactly satisfied when the ZMP is identical to the CP (equation (A.1)), or

$$
\left.\vec{\tau}_{\text {G.R. }}\left(\vec{r}_{Z M P}\right)\right|_{\text {horizontal }}=-\int_{g s b}\left(\vec{r} \times \frac{\vec{g}}{g}\right) d F_{G . R . Z}+\frac{\int_{g s b} \vec{r} d F_{G . R . Z}}{\int_{g s b} d F_{G . R . Z}} \times \frac{\vec{g}}{g} \int_{g s b} d F_{G . R . Z}=0 \quad Q E D^{\circ}
$$

Hence, for a flat horizontal support base, the ZMP and the CP exactly coincide.

\section{The Uniqueness of the ZMP and the CP for Complex Contact Topologies}

Consider the human model shown in Figure 7. Here the model's hand and foot are exerting forces against a non-horizontal contact surface. Given the net CM force, the ground reaction force may be obtained by simply subtracting the gravitational force. Given the CM location and the net moment about the CM, the ZMP line may be constructed. The intersection of that line with the contact surface then defines the ZMP location. In distinction, the CP may be obtained by integrating across the contact surface according to the first equality of equation (A.1). Hence, the $\mathrm{CP}$ can be positioned anywhere inside the convex hull represented by a 3-D, CP embedding volume and encompassing the contact foot and contact hand. For this particular example, the $\mathrm{CP}$ is not a ground reference point at all but is located above the contact surface.

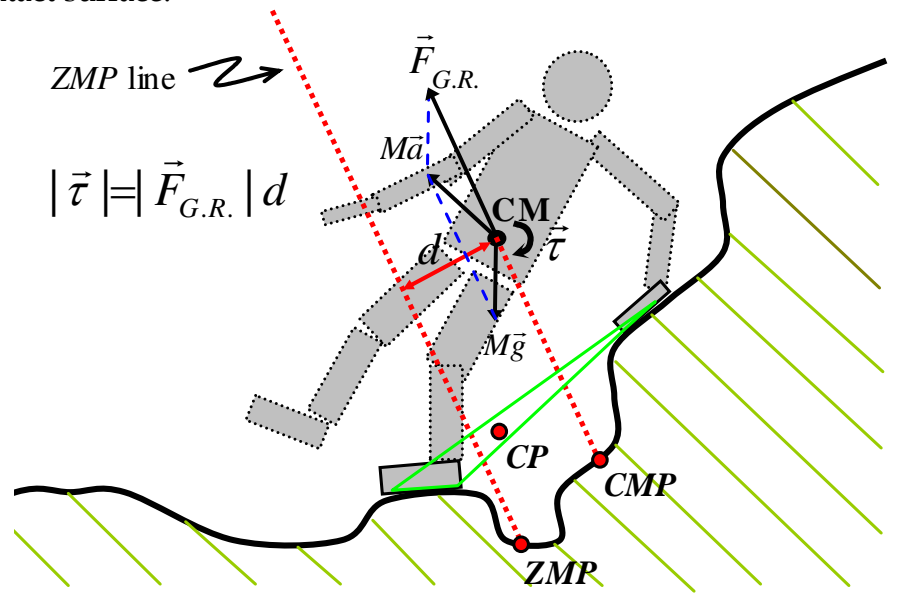

Fig. 7. Dynamical multi-link humanoid model with hand and foot contact. The ground reaction force originates at the ZMP. Inertia and the force of gravity originate at the $\mathrm{CM}$ point. As is shown in the figure, the ZMP and the CP point do not coincide for nonhorizontal contact surfaces. 
Using mathematical notation, we show that the ZMP is not equal to the $\mathrm{CP}$ for nonhorizontal contact surfaces like those depicted in Figure 8. For a general distribution of a normal unit vector field, $\vec{n}_{\perp}(\vec{r}) \neq \overrightarrow{\text { const. }}$, defined on all contact surfaces, one may show that the $\mathrm{ZMP}$ is not equal to the $\mathrm{CP}$ by first defining the $\mathrm{ZMP}$ as

$$
\left[\int_{s b}\left(\vec{r}-\vec{r}_{C M}\right) \times d \vec{F}_{R .}\right]_{h o r .}=\left[\left(\vec{r}_{Z M P}-\vec{r}_{C M}\right) \times \int_{s b} d \vec{F}_{R .}\right]_{h o r}
$$

where $\vec{r}_{Z M P}$ is on the external contact surface. One can then set this definition of the ZMP equal to the $\mathrm{CP}$ (equation (A.1)), to observe that

$$
\int_{s b}\left(\vec{r} \times d \vec{F}_{R}\right)_{h o r .} \neq\left[\frac{\int_{s b} \vec{r}\left(d \vec{F}_{R .} \cdot \vec{n}\right)}{\int_{s b}\left(d \vec{F}_{R .} \cdot \vec{n}\right)} \times \int_{s b} d \vec{F}_{R .} .\right.
$$

Hence, the ZMP and the $\mathrm{CP}$ do not always coincide and should therefore not be considered identical physical quantities. It should be noted that for expressions (A.6) and (A.7) we avoided the prefix ground to stress that any type of external contact surface is permissible when the ZMP is defined according to equation (A.6). Also, using this formalization, any body segment may be in contact with the external surface.

\section{Acknowledgments}

The authors wish to thank Paolo Bonato and Jennifer Lelas at the Spaulding Rehabilitation Hospital Gait Laboratory, Boston, Massachusetts for their helpful suggestions and support. The authors also thank Waleed Farahat, Andreas Hofmann, Dan Paluska, Ben Swilling and Russ Tedrake for their useful suggestions and technical support.

\section{Symbol Table (in alphabetical order)}

$\vec{a}_{i}$

$\alpha$

$D$

$D_{A}$

$D_{R}$

$D_{S}$

$D_{S}{ }^{i}$

$\vec{F}$

$\vec{F}_{\text {ankle }}$
Body segment $i$ center of mass acceleration.

Parameter used for optimization of human model mass parameters.

The relative mass distribution described by a 16component vector.

The average relative mass distribution (Winter 1990).

The resulting relative mass distribution.

The subject specific relative mass distribution obtained by equal density assumption.

The relative mass of the i-th link.

Net force acting on a whole body (in free fall $\left.F_{x}=F_{y}=0, \quad F_{z}=-M g\right)$.

The net force at the stance foot ankle joint exerted from the rest of the body. 


\section{Ground reaction force.}

The component of the ground reaction force normal to the surface.

Reaction force (general surface).

Net zero force in $\mathrm{x}$ direction corresponding to the Moment Balance Strategy.

Net zero force in $\mathrm{x}$ direction corresponding to the ZeroMoment Balance Strategy.

Gravitational constant $\left(9.81 \mathrm{~m} / \mathrm{s}^{2}\right)$.

Gravitational vector $\left(-g \cdot \vec{e}_{Z}\right)$.

Body segment $i$ inertia tensor about the link's center of mass.

The time dependent segment $i$ moment of inertia tensor about the center of mass.

The time dependent whole body moment of inertia tensor about the center of mass.

The desired whole body angular momentum.

Body mass.

Body segment $i$ mass.

The unit vector normal to the surface and pointing away from the ground.

The average distribution of link densities (Winter 1990).

Density profile described by the 16-component vector.

Density of the i-th link.

The pressure at location $\vec{r}$.

Body center of mass.

Centroidal Moment Pivot point.

Body segment $i$ center of mass.

Zero Moment Point.

Time.

Horizontal component (orthogonal to gravity vector) of the net moment about the Zero Moment Point.

The desired target whole body moment about the center of mass.

The resulting moment exerted from the ground on the body about the origin of the lab reference frame.

Horizontal component (orthogonal to gravity vector) of the moment of ground reaction force about the Zero Moment Point. 


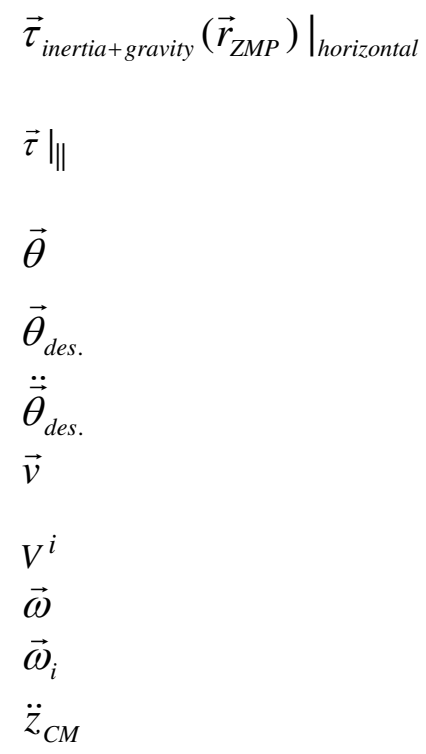

Horizontal component (orthogonal to gravity vector) of the moment due to inertial and gravitational forces about the Zero Moment Point.

The component of the whole body moment parallel to the flat surface (i.e. $\left.\vec{\tau}\right|_{\|} \cdot \vec{n}_{\perp}=0$ ).

The time dependent whole body angular excursion vector.

The desired target whole body angular excursion.

The desired target whole body angular acceleration.

The time dependent whole body center of mass velocity. (error in manuscript)

The volume of the i-th link.

The time dependent whole body angular velocity vector.

Body segment $i$ angular velocity.

Body center of mass acceleration in the vertical direction (in free fall $\ddot{z}_{C M}=-g$ ).

\section{References}

Ankrah, S. and Mills, N.J. 2003. Analysis of ankle protection in football. Sports Engineering 6.

Arakawa, T. and Fukuda, T. 1997. Natural motion generation of biped locomotion robot using hierarchical trajectory generation method consisting of GA, EP layers. Proceedings of the IEEE International Conference on Robotics and Automation, pp. 211216.

Borelli, G. A. 1680. De Motu Animalium (English) Translated by P. Maquet, Springer-Verlag, Berlin, Heidelberg, 1989.

Braune, W. and Fischer, O. 1895. Der Gang des Menschen. I Theil. Abh. K. Sachs. Ges. Wiss. Math.-Phys. Classe, 21:153.

Chew, C., Pratt, J. and Pratt, G. 1999. Blind walking of a planar bipedal robot on sloped terrains. IEEE International Conference on Robotics and Animation. Detroit, Michigan.

Darling, D. 2002. The Complete Book of Spaceflight: From Apollo 1 to Zero Gravity. John Wiley \& Sons, New York.

Dapena, J. and McDonald, C. 1989. A three-dimensional analysis of angular momentum in the hammer throw. Med. Sci. in Sports Exerc. 21: 206-220.

Dasgupta, A. and Nakamura, Y. 1999. Making feasible walking motion of humanoid robots from human motion capture data. Proceedings of the IEEE International Conference on Robotics and Automation, Detroit, MI, U.S.A., pp. 47-52.

Elftman, H. and Manter, J. 1934. A cinematic study of the distribution of pressure in the human foot. Science. 80: 484.

Elftman, H 1938. The measurement of the external force in walking. Science. 88: 152-153.

Elftman, H. (1939). The function of the arms in walking. Human Biology. 11: 529-535.

Full, B. and Koditschek, D. 1999. Templates and Anchors: Neural Mechanical Hypotheses of Legged Locomotion on Land. J. Exp. Biol. 202: 3325-3332. 
Goswami, A. 1999. Postural stability of biped robots and the foot-rotation indicator (FRI) point. International Journal of Robotics Research 18(6): 523-533.

Goswami, A. and Kallem, V. 2004. Rate of change of angular momentum and balance maintenance of biped robots. Proceedings of the IEEE International Conference on Robotics and Automation, New Orleans, LA, U.S.A., pp. 3785-3790.

$\mathrm{Gu}$, W. 2003. The Regulation of Angular Momentum During Human Walking. Undergraduate Thesis, Massachusetts Institute of Technology, Physics Department.

Herr, H., Whiteley, G.P. and Childress, D. 2003. Cyborg Technology--Biomimetic Orthotic and Prosthetic Technology. Biologically Inspired Intelligent Robots, Y. Bar-Cohen and C. Breazeal, Bellingham, Washington, SPIE Press, pp. 103-143.

Herr, H., Hofmann, A., Popovic, M. 2003. New Horizons for Orthotic \& Prosthetic Technology: Merging Body and Machine. Presented at the ZIF International Conference on Walking Machines, Bielefeld, Germany.

Herr, H. and Popovic, M. 2004. Angular momentum regulation in human walking. J. Exp. Biol. (In press).

Hinrichs, R., Cavanagh, P. and Williams, K. (1983). Upper extremity contributions to angular momentum in running. Biomechanics VIII-B. Champaign, IL:Human Kinetics. pp. 641-647.

Hirai K. 1997. Current and Future Prospective of Honda Humanoid Robot. Proceedings of the IEEE/RSJ International Conference on Intelligent Robots and Systems, Grenoble, France, pp. 500-508.

Hirai, K., Hirose, M., Haikawa Y. and Takenaka T. 1998. The Development of Honda Humanoid Robot. Proceedings of the IEEE International Conference on Robotics and Automation, Leuven, Belgium, pp. 1321-1326.

Hofmann, A., Popovic, M., Massaquoi, S. and Herr, H. 2004. A Sliding Controller for Bipedal Balancing Using Integrated Movement of Contact and Non-Contact Limbs. Proceedings of the IEEE/RSJ International Conference on Intelligent Robots and Systems, Sendai, Japan.

Hofmann, A. 2003. Control Rules for Biomimetic Human Bipedal Locomotion Based on Biomechanical Principles. Ph.D. Thesis Proposal, Submitted to the Computer Science and Electrical Engineering Department, Massachusetts Institute of Technology.

Huang, Q., Yokoi, K., Kajita, S., Kaneko, K., Aria, H., Koyachi, N. and Tanie, K. 2001. Planning walking patterns for a biped robot. IEEE Transactions on Robotics and Automation, vol. 17:(3)280-289.

Kagami, S., Kanehiro, F., Tamiya, Y., Inaba, M. and Inoue, H. 2000. Autobalancer: An online dynamic balance compensation scheme for humanoid robots. Proceedings of the $4^{\text {th }}$ International Workshop on Algorithmic Foundations, WAFR '00, pp. SA-79-SA-89.

Kajita, S., Kanehiro, F., Kaneko, K., Fujiwara, K., Harada, K., Yokoi, K. and Hirukawa, H. 2003. Resolved Momentum Control: Humanoid Motion Planning based on the Linear and Angular Momentum. Proceedings of the IEEE/RSJ International Conference on Intelligent Robots and Systems, Las Vegas, NV, U.S.A., pp. 1644-1650.

Kajita, S., Nagasaki, T., Kaneko, K., Yokoi, K. and Tanie, K. 2004. A Hop towards Running Humanoid Biped. Proceedings of the IEEE International Conference on Robotics and Automation, New Orleans, LA, U.S.A., pp. 629-635.

Kondak, K. and Hommel, G. 2003. Control and online computation of stable movement for biped robots. Proc. International Conference on Intelligent Robots and Systems (IROS). Las Vegas, NV, USA. 
LeBlanc, M. and Dapena, J. 1996. Generation and transfer of angular momentum in the javelin throw. In Proceedings of the 20th annual meeting of the American Society of Biomechanics. Atlanta, Georgia, pp.17-19.

Li, Q., Takanishi, A. and Kato, I. 1993. Learning control for a biped walking robot with a trunk. Proceedings of the IEEE/RSJ International Conference on Intelligent Robots and Systems, Yokohama, Japan, pp. 1771-1777.

Popovic, M., Gu, W. and Herr, H. 2002. Conservation of Angular Momentum in Human Movement. MIT AI Laboratory-Research Abstracts, September 2002. pp. 264-265.

Popovic M., and Herr, H. 2003. Conservation of whole body angular momentum. Presented at the Neuro-Muscular Research Center Seminar Series. March 20, Boston University, Boston, Massachusetts, USA.

Popovic, M., Hofmann, A. and Herr, H. 2004a. Angular Momentum Regulation during human walking: Biomechanics and Control. Proceedings of the IEEE International Conference on Robotics and Automation, New Orleans, LA, U.S.A., pp. 2405-2411.

Popovic, M., Englehart, A. and Herr, H. 2004. Angular Momentum Primitives for Human Walking: Biomechanics and Control. Proceedings of the IEEE/RSJ International Conference on Intelligent Robots and Systems, Sendai, Japan., pp. 1685-1691.

Popovic, M., Hofmann, A. and Herr, H. 2004b. Zero spin angular momentum control: definition and applicability. Proceedings of the IEEE-RAS/RSJ International Conference on Humanoid Robots, Los Angeles, CA.

Popovic, M. and Herr, H. 2005. Global Motion Control and Support Base Planning. Proceedings of the IEEE/RSJ International Conference on Intelligent Robots and Systems, Alberta, Canada.

Popovic, M., Goswami, A. and Herr, H. 2005. Ground Reference Points in Legged Locomotion: Definitions, Biological Trajectories and Control Implications. International Journal of Robotics Research 24(12): 1013-1032.

Pratt, G. 2002. Low Impedance Walking Robots. Integ. and Comp. Biol. 42:174-181.

Raibert, M. 1986. Legged Robots that Balance. The MIT Press, Cambridge Massachussetts.

Rose, J. and Gamble, J. G. 1994. Human Walking. 2nd edition. Baltimore, Maryland: Williams and Wilkins.

Sardain, P. and Bessonnet, G. 2004. Forces Acting on a Biped Robot. Center of PressureZero Moment Point. IEEE Transactions on Systems, Man and Cybernetics, Part A, 34(5): 630- 637.

Schaal, S. 1999. Is imitation learning the route to humanoid robots? Trends in Cognitive Sciences. 3: 233-242.

Shih, C. L., Li, Y. Z., Churng, S., Lee, T. T. and Gruver W. A. 1990. Trajectory synthesis and physical admissibility for a biped robot during the single-support phase. Proceedings of the IEEE/RSJ International Conference on Intelligent Robots and Systems, pp. 1646-1652.

Shih, C. L. 1996. The dynamics and control of a biped walking robot with seven degrees of freedom. ASME Journal of Dynamic Systems, Measurement and Control. 118: 683-690.

Takanishi, A., Ishida, M. Yamazaki, Y. and Kato, I. 1985. The realization of dynamic walking by the biped robot WL-10RD. Proceedings of the International Conference on Advanced Robotics, Tokyo, Japan, pp. 459-466.

Takanishi, A., Lim, H.-ok, Tsuda, M. and Kato, I. 1990. Realization of Dynamic Biped Walking Stabilized by Trunk Motion on a Sagitally Uneven Surface. Proceedings of the IEEE/RSJ International Conference on Intelligent Robots and Systems, pp. 323-330. 
Tilley A. R. and Dreyfuss H. 1993. The measure of man and woman. New York, Whitney Library of Design, an imprint of Watson-Guptill Publications.

Vukobratovic, M. and Juricic, D. 1969. Contributions to the synthesis of biped gait. IEEE Transactions on Biomedical Engineering, BME-16, pp. 1-6.

Vukobratovic, M., Frank, A., and Juricic, D. 1970. On the stability of biped locomotion. IEEE Transactions on Biomedical Engineering, BME-17(1): 25-36.

Vukobratovic, M. and Stepanenko, J. 1972. On the Stability of Anthropomorphic System. Mathematical Biosciences 15:1-37.

Vukobratovic, M., Stepanenko, Y. U. 1973. Mathematical models of general anthropomorphic systems. Mathematical Biosciences. 17: 191-242.

Vukobratovic, M. and Borovac, B. 2004. Zero-moment point - thirty five years of its life. International Journal of Humanoid Robotics. 1(1): 157-173.

Winter, D. A. 1990. Biomechanics and Motor Control of Human Movement. New York, John Wiley \& Sons, Inc.

Witkin, A. and Kass, M. 1988. Spacetime constraints. In John Dill, editor, Computer Graphics (SIGGRAPH '88 Proceedings). 22: 159-168.

Yamaguchi, J., Soga, E., Inoue, S. and Takanishi, A., 1999. Development of a Bipedal Humanoid Robot - Control Method of Whole Body Cooperative Dynamic Biped Walking. Proceedings of the IEEE International Conference on Robotics and Automation, pp. 368-374.

Yamaguchi, J., Takanishi, A. and Kato, I. 1993. Development of a biped walking robot compensation for three-axis moment by trunk motion. Proceedings of the IEEE/RSJ International Conference on Intelligent Robots and Systems, Yokohama, Japan. 


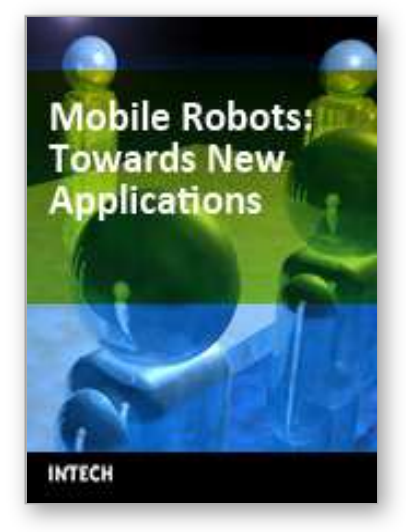

\author{
Mobile Robots: towards New Applications \\ Edited by Aleksandar Lazinica
}

ISBN 978-3-86611-314-5

Hard cover, 600 pages

Publisher I-Tech Education and Publishing

Published online 01, December, 2006

Published in print edition December, 2006

The range of potential applications for mobile robots is enormous. It includes agricultural robotics applications, routine material transport in factories, warehouses, office buildings and hospitals, indoor and outdoor security patrols, inventory verification, hazardous material handling, hazardous site cleanup, underwater applications, and numerous military applications. This book is the result of inspirations and contributions from many researchers worldwide. It presents a collection of wide range research results of robotics scientific community. Various aspects of current research in new robotics research areas and disciplines are explored and discussed. It is divided in three main parts covering different research areas: Humanoid Robots, Human-Robot Interaction, and Special Applications. We hope that you will find a lot of useful information in this book, which will help you in performing your research or fire your interests to start performing research in some of the cutting edge research fields mentioned in the book.

\title{
How to reference
}

In order to correctly reference this scholarly work, feel free to copy and paste the following:

Marko B. Popovic and Hugh Herr (2006). Ground Reference Points in Legged Locomotion: Definitions, Biological Trajectories and Control Implications, Mobile Robots: towards New Applications, Aleksandar Lazinica (Ed.), ISBN: 978-3-86611-314-5, InTech, Available from:

http://www.intechopen.com/books/mobile_robots_towards_new_applications/ground_reference_points_in_legg ed_locomotion_definitions_biological_trajectories_and_control_impli

\section{INTECH}

open science | open minds

\section{InTech Europe}

University Campus STeP Ri

Slavka Krautzeka 83/A

51000 Rijeka, Croatia

Phone: +385 (51) 770447

Fax: +385 (51) 686166

www.intechopen.com

\section{InTech China}

Unit 405, Office Block, Hotel Equatorial Shanghai

No.65, Yan An Road (West), Shanghai, 200040, China

中国上海市延安西路65号上海国际贵都大饭店办公楼 405 单元

Phone: +86-21-62489820

Fax: $+86-21-62489821$ 
(C) 2006 The Author(s). Licensee IntechOpen. This chapter is distributed under the terms of the Creative Commons Attribution-NonCommercialShareAlike-3.0 License, which permits use, distribution and reproduction for non-commercial purposes, provided the original is properly cited and derivative works building on this content are distributed under the same license. 